\title{
Ground State Properties of Cold Bosonic Atoms At Large Scattering Lengths
}

\author{
Jun Liang Song and Fei Zhou \\ Department of Physics and Astronomy, The University of British Columbia, Vancouver, B. C., Canada V6T1Z1
}

(Dated: October 26, 2018)

\begin{abstract}
In this Letter, we study bosonic atoms at large scattering lengths using a variational method where the condensate amplitude is a variational parameter. We further examine momentum distribution functions, chemical potentials, the speed of sound, and spatial density profiles of cold bosonic atoms in a trap in this limit. The later two properties turn out to bear similarities of those of Fermi gases. The estimates obtained here are applicable near Feshbach resonances, particularly when the fraction of atoms forming three-body structures is small and can be tested in future cold atom experiments.
\end{abstract}

Bose-Einstein condensates(BECs) near Feshbach resonances have been one of the most exciting ultracold systems studied so far in experiments $1,2,2,3,4,5,6$, 7, 8, 9]. On one side of the resonances where the scattering lengths are negative, fascinating collapse-growth cycles due to thermal clouds and spectacular controlled collapsing-exploding dynamics have been observed 1, 2, 3] and studied theoretically [4]. On the other side, towards the resonances where scattering lengths are positive, strongly repulsive ultracold bosonic atoms and their intriguing properties have been explored [5, 6, 6, 7, 8]. Despite the reduced lifetime of the cold gases in this limit due to enhanced three-body recombination, quite remarkable progress has been made to probe interactions between atoms. Recently, pursuit in this direction has been revived, and more vigorous efforts have been made [8, 9]. Our theoretical studies in this Letter are mainly motivated by these experiments. Bose gases at large positive scattering lengths have been a horrendously challenging topic in theoretical physics for more than half a century 10, 11, 12, 13, 14]. The standard low density expansion that works quite well for dilute gases is not applicable when the scattering length $a$ is comparable to or even larger than the mean interparticle distance $d$. Here we suggest a variational approach which takes into account two-body correlations and can be extended to the limit of a large positive scattering length. We further apply this approach to estimate various fundamental properties of cold bosonic atoms near Feshbach resonances, particularly when the fraction of atoms forming three-body structures is small. Unique features in the momentum distribution function, chemical potential, speed of sound and the cold atom density profile in a trap can be potentially probed in experiments.

Cold bosonic atoms at large scattering lengths were also previously addressed in a few inspiring theoretical papers [15, 16]. Cowell et al. estimated chemical potentials and condensate fractions by employing distinctly different Jastrow wavefunctions [15]. There are a few interconnected differences between their results and ours. First, while the physics at distances much shorter than the mean interparticle distance $d$ is described quite accurately by the Jastrow wave functions, basic aspects of the long wavelength physics are not expected to be well captured. On the other hand, our wave function is constructed under a constraint in Eq.(2) and captures essential features of low energy collective properties of BECs. For instance, the momentum distribution function $n_{\mathbf{k}}$ has a $\frac{1}{k}$ divergence near $k=0$ for all scattering lengths, and at short distances our wave function is almost identical to the solution to the Schrödinger equation for two interacting atoms. Second, since the contribution to the depletion fraction, or the fraction of atoms occupying nonzero momentum states, is mainly from low energy states, we expect that our results are more reliable. In fact, we find that the depletion fraction reaches a constant value of about 0.5 near resonances. On the contrary, the condensate fraction estimated in Ref. 15] quickly reaches zero when the scattering length $a$ becomes comparable to $d$, suggesting that atoms could be completely depleted from the zero momentum state and there should be an unexpected quantum phase transition at a finite scattering length. Third, chemical potentials near resonances estimated there appear to be bigger than the values obtained in our calculations. This seems to imply that the trial wave functions adopted here should be an energetically better candidate for ground states.

The trial wave function in Eq.(3) effectively encodes two-body correlations. To include high-order correlations such as three-body effects, a much more sophistic ansatz is needed. A nontrivial role of three-body interactions was previously appreciated by Braaten et al. in Ref.[16], where the effects on BECs were estimated in the limit of a small scattering length. Although there was no definite evidence of Efimov trimers in BECs of sodium or rubidium atoms studied in Ref. [5, [6, 7, 8], an earlier experiment on relaxation rates of cesium atoms did show, as a precursor of two-body resonances, additional structures which had been attributed to Efimov states [17, 18, 19, 20]. More efforts are to be made to understand the nature of BECs in this limit and the approach proposed below is a baby step towards this direction. Our results are valid when the three-body correlations induced by Efimov trimers are not dominating. The question of whether the emergence of Efimov trimers introduces distinct modulations to the scaling functions discussed below, or mainly sets the lifetime of BECs, represents an exciting new direction that is worth pursuing. 
Moreover, our scaling hypothesis works best when the typical range of interactions $r_{0}$ is much less than the inter-particle distance $d$. When the density increases, deviations from the scaling behaviors become substantial, and the scaling functions proposed below are no longer sufficient for characterizing BECs. Eventually, a quantum gas might undergo a transition to a dense liquid phase when $r_{0}$ becomes comparable to $d$. For cold atoms, this fortunately only occurs at a density which is not experimentally accessible because of severe trap losses.

We consider bosonic atoms that interact with a short range potential of range $r_{0}$ and scatter at two-body scattering lengths $a(>0)$. For BECs with a number density $\rho_{0}$, assuming two-body effects are dominating, we can generally express the momentum distribution function $n_{\mathbf{k}}$ and the chemical potential $\mu$ in terms of dimensionless functions $f$ and $h$, i.e., $n_{\mathbf{k}}=f\left(k d, \frac{a}{d}, \frac{r_{0}}{d}\right), \mu=\epsilon_{F} h\left(\frac{a}{d}, \frac{r_{0}}{d}\right)$; and $d=\left(\frac{3}{4 \pi \rho_{0}}\right)^{1 / 3}, \epsilon_{F}=\frac{\left(6 \pi^{2} \rho_{0}\right)^{2 / 3}}{2 m}$. For short range interactions, $r_{0}$ is much smaller than the mean inter-particle distance $d$ so that we approximate $\frac{r_{0}}{d}$ to be zero but $a$ can vary over a range from much smaller than $d$ to much bigger than $d$. Function $f$ and $h$ thus depend only on two dimensionless variables $x=k d$ and $y=\frac{a}{d}$ and are reduced to two scaling functions $f(x, y)$ and $h(y)$, respectively. The functional form of $f(x, y)$ and $h(y)$ proposed in this way does not depend on details of interaction potentials or number densities or scattering lengths and is universal; $f$ and $h$ characterize basic properties of BECs. Note that, as illustrated below, the Fermi energy $\epsilon_{F}$ that is normally defined for a Fermi gas with the same number density $\rho_{0}$ turns out to be the only relevant energy scale for BECs near resonances.

When $a$ is much smaller than $d$, these functions can be obtained by using the standard mean field [11, 12, 13, 14]. Indeed, in the dilute limit when $y$ is much less than unity one can verify that

$$
\begin{aligned}
& f(x, y)=\frac{1}{2}\left(\frac{x^{2}+6 y}{\sqrt{x^{2}\left(x^{2}+12 y\right)}}-1\right), \\
& h(y)=\left(\frac{32}{3 \pi^{2}}\right)^{1 / 3} y, \quad g(y)=\frac{4}{\sqrt{3} \pi} y^{3 / 2},
\end{aligned}
$$

where we also introduce $g(y)$ for the depletion fraction. $f(x, y)$ is divergent as $\sqrt{y} / x$ when $x$ or momentum $k$ approaches zero; this behavior is an indication of gapless soundlike collective excitations in BECs. Furthermore, that $f(x, y)$ decays as $y^{2} / x^{4}$ in the large- $x$ or large- $k$ limit reflects the free particle nature of high energy excitations. For cold atoms at large scattering lengths, $y$ is substantial and the form of $f$ and $h$ functions remains to be understood. In the following we are going to investigate these scaling functions in the limit when $a$ (or $y$ ) becomes comparable to or bigger than $d$ (or 1$)$.

To quantitatively study $f$ and $h$ functions in the limit of a large scattering length, we adopt a variational approach to BECs. In this method, $c_{0}$, the condensate am- plitude and $g_{\mathbf{k}}, \mathbf{k} \neq 0$, the pairing amplitude that is related to the occupation number of atoms in a state of momentum $\mathbf{k}$, are variational parameters. We then minimize the energy with respect to $g_{\mathbf{k}}$ and $c_{0}$ but with the total number of atoms $N_{T}$ fixed.

To introduce trial wave functions which are viable in both small and large scattering-length limits, we require that at any given scattering length the ground state should be a vacuum of Bogoliubov quasi-particles and is annihilated by a set of quasiparticle operators

$$
\left.\left(\frac{1}{\sqrt{1-\left|g_{\mathbf{k}}\right|^{2}}} \hat{a}_{\mathbf{k}}-\frac{g_{\mathbf{k}}}{\sqrt{1-\left|g_{\mathbf{k}}\right|^{2}}} \hat{a}_{-\mathbf{k}}^{\dagger}\right) \mid \text { g.s. }\right\rangle=0 .
$$

Here $\hat{a}_{\mathbf{k}}\left(\hat{a}_{\mathbf{k}}^{\dagger}\right)$ is an annihilation (creation) operator for an atom with momentum $\mathbf{k}$. Detailed structures of the quasi-particle operators are specified by real variables $g_{\mathbf{k}}$ and will be determined variationally below. The ansatz that satisfies Eq.(2) can be written as

$$
\mid \text { g.s. }\rangle=\mathcal{A}^{-1 / 2} \exp \left(c_{0} \hat{a}_{0}^{\dagger}\right) \prod_{\mathbf{k} \cdot \hat{\mathbf{z}}>\mathbf{0}} \exp \left(g_{\mathbf{k}} \hat{a}_{\mathbf{k}}^{\dagger} \hat{a}_{-\mathbf{k}}^{\dagger}\right)|0\rangle .
$$

Here $\mathcal{A}$ is the normalization factor. Again $c_{0}$ is the condensation amplitude and $g_{\mathbf{k}}$ is the pairing amplitude with $\left|g_{\mathbf{k}}\right|<1$; for ground states, we further assume $g_{-\mathbf{k}}=g_{\mathbf{k}}$. This trial wave function encodes two-body correlations but not three-body ones. Similar wave functions have been used to study pair condensates of attractive bosons 21]. $n_{\mathbf{k}}$, the occupation number of atoms with momentum $\mathbf{k}$, is a simple function of $g_{\mathbf{k}}$

$$
n_{\mathbf{k}}=\left\langle\hat{a}_{\mathbf{k}}^{\dagger} \hat{a}_{\mathbf{k}}\right\rangle=\frac{\left|g_{\mathbf{k}}\right|^{2}}{1-\left|g_{\mathbf{k}}\right|^{2}} .
$$

The Hamiltonian of cold bosons is

$$
H=\sum_{\mathbf{k}} \epsilon_{\mathbf{k}} \hat{a}_{\mathbf{k}}^{\dagger} \hat{a}_{\mathbf{k}}+\frac{1}{2} \sum_{\mathbf{k}_{1}, \mathbf{k}_{2}, \mathbf{q}} \hat{a}_{\mathbf{k}_{1}+\mathbf{q}}^{\dagger} \hat{a}_{\mathbf{k}_{\mathbf{2}}-\mathbf{q}}^{\dagger} U(\mathbf{q}) \hat{a}_{\mathbf{k}_{1}} \hat{a}_{\mathbf{k}_{2}}
$$

$U(\mathbf{q})=\frac{1}{\Omega} \int d^{3} r U(\mathbf{r}) \exp (i \mathbf{q} \cdot \mathbf{r})$ is a two-body interaction potential, and $\Omega$ is the volume of the system. The total energy $E_{T}$ of the trial state $\mid$ g.s. $>$ is evaluated to be

$$
\begin{aligned}
E_{T} & =\sum_{\mathbf{k}} \epsilon_{\mathbf{k}} \frac{\left|g_{\mathbf{k}}\right|^{2}}{1-\left|g_{\mathbf{k}}\right|^{2}}+\frac{U(0)}{2}\left|c_{0}\right|^{4} \\
& +\sum_{\mathbf{k}, \mathbf{q} \neq \mathbf{0}} \frac{U(\mathbf{k}-\mathbf{q})+U(0)}{2} \frac{\left|g_{\mathbf{k}}\right|^{2}}{1-\left|g_{\mathbf{k}}\right|^{2}} \frac{\left|g_{\mathbf{q}}\right|^{2}}{1-\left|g_{\mathbf{q}}\right|^{2}} \\
& +\sum_{\mathbf{k}, \mathbf{q} \neq \mathbf{0}} \frac{U(\mathbf{k}-\mathbf{q})}{2} \frac{g_{\mathbf{q}}^{*}}{1-\left|g_{\mathbf{q}}\right|^{2}} \frac{g_{\mathbf{k}}}{1-\left|g_{\mathbf{k}}\right|^{2}} \\
& +\sum_{\mathbf{q} \neq \mathbf{0}} \frac{U(\mathbf{q})+U(0)}{2} \frac{2\left|g_{\mathbf{q}}\right|^{2}}{1-\left|g_{\mathbf{q}}\right|^{2}}\left|c_{0}\right|^{2} \\
& +\sum_{\mathbf{q} \neq \mathbf{0}} \frac{U(\mathbf{q})}{2} \frac{c_{0}^{2} g_{\mathbf{q}}^{*}+c_{0}^{* 2} g_{\mathbf{q}}}{1-\left|g_{\mathbf{q}}\right|^{2}} .
\end{aligned}
$$



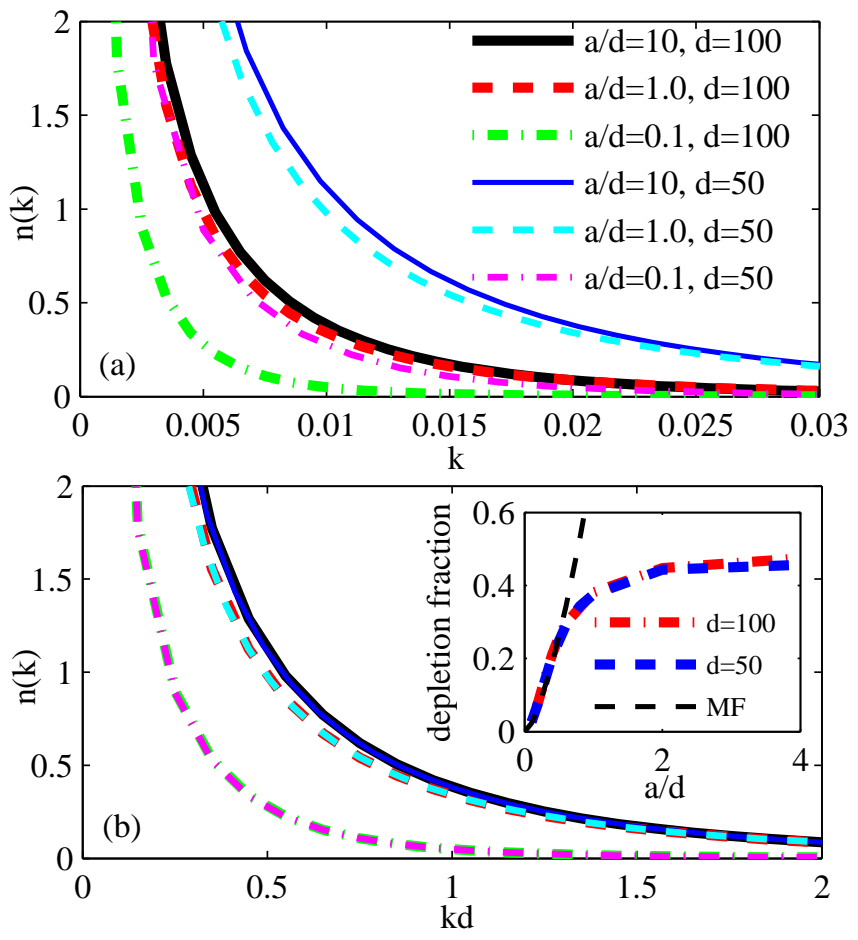

FIG. 1: (Color online) The momentum distribution function $n(k)$ for different interparticle distance $d$ and scattering length $a$ [in units of $r_{0}$, the range of interaction and in a, $k$ is in units of $\left.\hbar / r_{0}\right]$. In all cases, $n(k)$ has a desired $1 / k$ divergence when $k$ approaches zero. In $\mathrm{b}), n(k)$ functions for different $d$ but with the same value of $y=\frac{a}{d}$ are further shown to collapse to a single scaling function when plotted against $x=k d$. The resultant three curves are for $f(x, y)$ with $y=0.1,1$ and 10 (from bottom to top). Depletion fraction $g(y)$ is plotted in the inset; the mean field(MF) $g(y)$ in Eq.(1) is also shown as a reference.

To facilitate discussions on large scattering lengths, we assume that the interaction potential is a square well one, $U(r)=-U$ when $r<r_{0}$ but otherwise is zero. The corresponding $s$-wave scattering length is $a=r_{0}-$ $\tan \left(\sqrt{m U} r_{0}\right) / \sqrt{m U}$. We choose the depth of the potential $U$ to be $\pi / 2<\sqrt{m U} r_{0}<\pi$ so that $r_{0}<a<\infty$.

To obtain ground states, we minimize the total energy in Eq.(6) with respect to parameters $g_{\mathbf{k}}$ and $c_{0}$, subject to a constraint that the total number $N_{T}$ is fixed,

$$
N_{T}=\left|c_{0}\right|^{2}+\sum_{\mathbf{k} \neq 0} \frac{\left|g_{\mathbf{k}}\right|^{2}}{1-\left|g_{\mathbf{k}}\right|^{2}} .
$$

When the potential is weakly repulsive, we verify that the minimization does lead to the standard results for weakly interacting BECs, i.e. Eq.(1). For attractive potentials introduced above, the minimization is carried out numerically. When scattering lengths are positive, one of the energy minima turns out to be a collection of molecules as expected from a two-body consideration; in these molecular states, the condensate amplitude is found
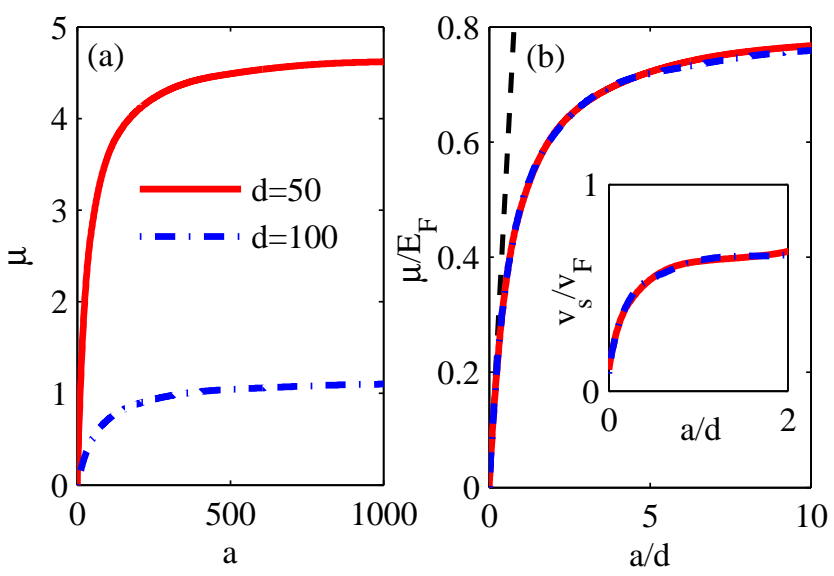

FIG. 2: (Color online) Chemical potentials $\mu$ and sound velocities $v_{s}$ as a function of scattering length. a) is for $\mu$ (in units of $1 / 2 m d^{2}, d=50$ versus $a$ ( $d$ and $a$ are in units of $\left.r_{0}\right)$; in b) we further plot $\mu$ in units of the Fermi energy $\epsilon_{F}$ as a function of $y=a / d$ and illustrate two plots in a) collapse into a single scaling curve. The resultant plot defines the scaling function $h(y)\left(=\mu / \epsilon_{F}\right)$ for an arbitrary $y$. The dashed line is for $h(y)$ in Eq.(1). Shown in the inset is $v_{s}$ (in units of the Fermi velocity $v_{F}$ ) versus $a / d$.

to be zero and $\left|g_{\mathbf{k}}\right|$ is less than unity for all $\mathbf{k}$. To understand BECs of scattering atoms in open or nonmolecular channels that are most relevant to experiments on cold atoms, we project away the molecular states and minimize the energy in the subspace of scattering channels. This is achieved by imposing a projection constraint on $g_{\mathbf{k}}, \sum_{\mathbf{k}} g_{\mathbf{k}}^{\text {mol }} g_{\mathbf{k}}^{*}=0$, where $g_{\mathbf{k}}^{\text {mol }}$ are the calculated values of $g_{\mathbf{k}}$ for molecular states. This vanishing inner product between molecular states and states of atoms effectively projects out a desired subspace of open channels.

Below we present results for BECs with different densities and scattering lengths. The minimization algorithm does converge leading to a ground state in the subspace when we set $g_{\mathbf{k}}+1$ to be proportional to $k$ in the close vicinity of $k=0[22]$. We further find that $g_{\mathbf{k}}$ decays as $\frac{1}{k^{2}}$ in the large- $k$ limit for all scattering lengths. Following the relation between $g_{\mathbf{k}}$ and $n_{\mathbf{k}}$ in Eq.(4), one then obtains the asymptotics of $n_{\mathbf{k}}$ in both large- $k$ and small- $k$ limits. The characteristics in these two limits are robust and, when the scattering length $a$ is tuned, remain the same as those in Eq.(1). However, the crossover energy between these two limits, which is approximately the chemical potential, strongly depends on the scattering lengths or densities (see Fig 11). When plotted against $x=k d$, data for $n_{\mathbf{k}}$ or $n(k)$ calculated for different densities and scattering lengths all collapse to a single set of curves which correspond to $f(x, y)$ for different $y=\frac{a}{d}$. Furthermore, we observe that the function $n(k)=f(x, y)$ quickly approaches $f_{\infty}(x)$ when $y$ exceeds unity. Using the momentum distribution function, we also estimate $g(y)$, the fraction of atoms that are depleted from the 

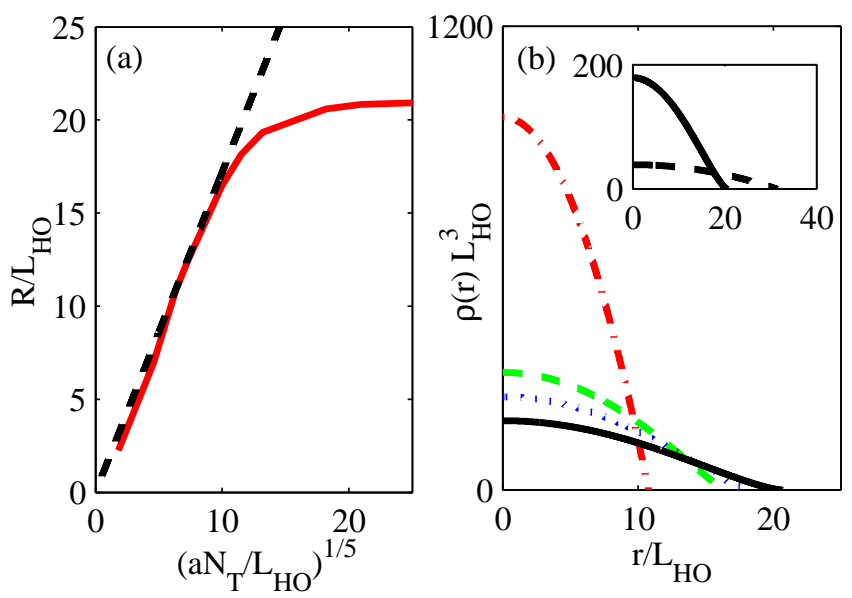

FIG. 3: (Color online) a) Radius $R$ of BECs in a spherical harmonic trap with harmonic length $L_{H O}$ as a function of scattering length $a$; the dashed line is the mean field Thomas-Fermi radius $R_{T F}$ (see discussions before Eq. (8) ). b)Spatial density profiles in a harmonic trap at different scattering lengths; the density at the center $\rho(0)$ is estimated to be $\rho(0) a^{3}=0.00012,0.038,0.24,180$ for the dasheddotted, dashed, dotted, and solid line respectively. In the inset, we also plot the mean field result(black dashed line) for $\rho(0) a^{3}=180$ for a comparison. In a), b), the total number of atoms is set to be $N_{T}=2 \times 10^{6}$.

zero momentum state; $g(y)$ saturates at a constant value near resonances.

The chemical potential is studied by evaluating $\mu=$ $\partial E_{T} / \partial N_{T}$. In the limit of large scattering length, the main characteristic is that $\mu$ saturates at a value of around $80 \%$ of the Fermi energy $\epsilon_{F}$ of the corresponding density. When the chemical potential in units of $\epsilon_{F}$ is plotted against scattering lengths $y=\frac{a}{d}$, all data again collapse to a single master curve which quantitatively defines the scaling function $h(y)\left(=\frac{\mu}{\epsilon_{F}}\right)$ introduced above; and $h(y)$ approaches 0.8 once $y$ becomes much bigger than unity (see Fig,2). $v_{s}$, the speed of sound that depends on the compressibility of BECs, can also be obtained by using the general relation $v_{s}^{2}=\rho_{0} / m\left(\partial \mu / \partial \rho_{0}\right)$.

The scattering-length dependence of the chemical potential discussed here implies a very peculiar evolution of sizes of BECs in a trap (with a harmonic length $L_{H O}$ ) when scattering lengths $a$ are increased. In the limit of a small scattering length the size of condensates increases as a function of scattering length $a$ and the mean field Thomas-Fermi radius in a spherical trap is $R_{T F} / L_{H O}=\left(15 N_{T} a / L_{H O}\right)^{1 / 5}[23]$. As the chemical potential saturates at a value of $0.8 \epsilon_{F}$ when scattering lengths become much larger than the typical interparticle distance in a trap, the radius of the BECs in this strongly interacting regime is also expected to approach a value of

$$
\frac{R}{L_{H O}}=A N_{T}^{1 / 6}
$$

Numerical calculations further show that $A=1.9$. As another application of our variational approach, we quantitatively investigate radii of BECs near resonances using a local density approximation (see Fig,3).

In conclusion, we have examined basic properties of cold bosonic atoms at large scattering lengths. Using the variational method, we estimate various properties that can be potentially tested in future cold atom experiments. Near resonances, we have found that the chemical potential, speed of sound, and the spatial density profile of cold bosons in a trap resemble the corresponding properties of Fermi gases. This particular aspect is also a unique feature of one-dimensional TonksGirardeau gases where bosons are viewed as fermionized particles [24, 25, 26]. Our results are applicable near Feshbach resonances but before the Efimov physics fully sets in. This work is supported by NSERC, the Canada and Canadian Institute for Advanced Research. We thank Jason T. L. Ho for a stimulating discussion.

[1] C. A. Sackett et al., Phys. Rev. Lett. 82, 876 (1999); C. C. Bradley et al., Phys. Rev. Lett. 75, 1687 (1995).

[2] E. A. Donley et al., Nature 412, 295 (2001).

[3] J. L. Roberts et al., Phys. Rev. Lett. 86, 4211 (2001).

[4] Y. Kagan et al., Phys. Rev. Lett. 79, 2604 (1997); M. Ueda et al., Phys. Rev. Lett. 80, 1576 (1998); R. A. Duine et al., Phys. Rev. Lett. 86, 2204 (2001).

[5] S. Inouye et al., Nature 392, 151 (1998).

[6] S. L. Cornish et al., Phys. Rev. Lett. 85, 1795 (2000).

[7] N. R. Claussen et al., Phys. Rev. Lett. 89, 010401(2002).

[8] S. B. Papp et al., Phys. Rev. Lett.101, 135301 (2008).

[9] S. E. Pollack et al., Phys. Rev. Lett.102, 090402(2009).

[10] N. N. Bogoliubov, J. Phys. USSR 11, 23 (1947).

[11] S. T. Beliaev, Sov. Phys. JETP. 7, 289 (1958).

[12] T. D. Lee, and C. N. Yang, Phys. Rev. 105, 1119 (1957).

[13] T. D. Lee, K. Huang, and C. N. Yang, Phys. Rev. 106, 1135 (1957).

[14] P. Nozieres and D. Pines, The theory of quantum liquids, Vol II Superfluid Bose Liquids, (Addison-Wesley, Redwood City, CA, 1990).

[15] S. Cowell et al., Phys. Rev. Lett. 88, 210403 (2002).

[16] E. Braaten et al., Phys. Rev. Lett. 88, 040401 (2002).

[17] V. Efimov, Phys. Lett. B. 33, 563 (1970); Sov. J. Nucl. Phys. 12, 589 (1971).

[18] T. Kraemer et al., Nature 440, 315-318 (2006).

[19] B. D. Esry et al., Phys. Rev. Lett. 83, 1751 (1999).

[20] F. D. Bedaque et al., Phys. Rev. Lett. 85, 908 (2000).

[21] P. Nozieres et al., J. Physique 43, 1133 (1982).

[22] This boundary condition equivalently requires that $n_{\mathbf{k}}$ diverge as $\frac{1}{k}$ near $k=0$, consistent with the long wave length physics discussed after Eq.(1).

[23] F. Dalfovo et al., Rev. Mod. Phys. 71, 463 (1999).

[24] M. Girardeau, J. Math. Phys. 1,516 (1960).

[25] B. Paredes et al., Nature 429, 277 (2004).

[26] T. Kinoshita et al., Science 305, 1125(2004). 